\title{
Inhibition of WNT/ $\beta$-catenin signaling under serum starvation and hypoxia induces adipocytic transdifferentiation in human leiomyoma cells
}

\author{
Hiroshi Harada ${ }^{1,2} \cdot$ Yojiro Tsuda $^{1} \cdot K^{2}$ Yabuki ${ }^{1} \cdot$ Eisuke Shiba $^{1} \cdot K^{2}$ Kazuyoshi Uchihashi ${ }^{3} \cdot$ Atsuji Matsuyama $^{1} \cdot$ \\ Yoshihisa Fujino $\mathbb{( i D}^{4} \cdot$ Toru Hachisuga $^{2} \cdot$ Masanori Hisaoka $^{1}$
}

Received: 31 August 2017 / Revised: 1 December 2017 / Accepted: 2 December 2017

(c) United States \& Canadian Academy of Pathology 2018

\begin{abstract}
Fatty metamorphosis is an uncommon alteration in uterine leiomyoma (i.e., lipoleiomyoma), and the pathogenetic mechanisms underlying this phenomenon remain poorly understood. Because a conditional deletion of $\beta$-catenin, a major transducer of the canonical Wingless/integrated (WNT) pathway, in the developing mouse uterus can induce adipogenesis in the myometrium, it is hypothesized that inhibition of the WNT/ $\beta$-catenin signaling may be also involved in the development of fat cells within uterine leiomyoma. In the current study, which was performed to address this point, intracytoplasmic lipid droplets were detectable in cultured human leiomyoma cells by treatment with a potent tankyrase inhibitor, XAV939, which antagonizes $\beta$-catenin, in a serum-starved culture medium without additional adipogenesis-inducing agents or supplements, and showed increasing accumulation in a time-dependent manner. In addition, the induction of fat cells was greatly enhanced under hypoxic conditions (i.e., 2.5\% $\mathrm{O}_{2}$ ) -recapitulating the local in vivo situation of uterine leiomyoma-in comparison to that under normoxic conditions (i.e., $21 \% \mathrm{O}_{2}$ ). The marker genes of differentiated fat cells such as ADIPOQ and PLIN were highly expressed in leiomyoma cells that were treated with XAV939 under hypoxia and serum starvation, whereas the immunohistochemical expression of desmin - a cytoskeletal protein representing smooth muscle differentiation-was downregulated, which appears in line with the switch in differentiation. The results of our study suggest that the inhibition of canonical WNT/ $\beta$-catenin signaling under the stress due to hypoxia and serum starvation can initiate adipocytic transdifferentiation or metaplasia in human uterine leiomyoma cells, which is potentially related to the development of lipoleiomyoma.
\end{abstract}

Electronic supplementary material The online version of this article (https://doi.org/10.1038/s41374-017-0020-5) contains supplementary material, which is available to authorized users.

Masanori Hisaoka

hisaoka@med.uoeh-u.ac.jp

1 Department of Pathology and Oncology, School of Medicine, University of Occupational and Environmental Health, Kitakyushu, Japan

2 Department of Obstetrics and Gynecology, School of Medicine, University of Occupational and Environmental Health, Kitakyushu, Japan

3 Department of Surgical Pathology, Kyushu Rosai Hospital, Kitakyushu, Japan

4 Department of Preventive Medicine and Community Health, School of Medicine, University of Occupational and Environmental Health, Kitakyushu, Japan
Smooth muscle cells, a major type of mesenchymal cells, are widely distributed throughout most of the human body and show remarkable plasticity. Their morphology and biological functions can change in response to a variety of patho-physiological conditions. Adipocytic transdifferentiation or metaplasia may be representative forms of such phenotypic plasticity. The phenomenon is most commonly encountered in neoplastic conditions of smooth muscle cells such as leiomyomas and angiomyomas [1-3]. However, the detailed mechanisms underlying adipocytic transdifferentiation remain largely unknown; however, the adipocytic differentiation of pluripotent mesenchymal cells in such lesions and the direct transformation of neoplastic smooth muscle cells are possible explanations [3-5].

Wingless/integrated (WNT) signaling is one of the representative and widely conserved intracellular signal pathways that have a pivotal role in various biological functions including cell differentiation, proliferation, 
polarity, and apoptosis in various organ systems. The canonical WNT signaling exerted by its major transducer, $\beta$-catenin, has been shown to inhibit adipogenesis $[6,7]$. In addition, a conditional deletion of $\beta$-catenin in the mesenchyme of the developing mouse uterus has been shown to induce adipogenesis in the myometrium [8]. These observations prompted us to investigate the biological role of the WNT/ $\beta$-catenin signaling, which is potentially involved in the above-mentioned plasticity of neoplastic smooth muscle cells (namely adipocytic transdifferentiation) in uterine leiomyoma.

Our study demonstrates that the adipocytic transdifferentiation of uterine leiomyoma cells can be induced by the inhibition of the canonical WNT/ $\beta$-catenin signaling using a tankyrase inhibitor (XAV939) that is known to stabilize axin, leading to the degradation of $\beta$-catenin [9], together with serum starvation. We also found that hypoxic conditions, to which uterine leiomyoma is understandably exposed in vivo [10], can further enhance the XAV939/ serum starvation-induced adipocytic transdifferentiation in leiomyoma cells.

\section{Materials and methods}

\section{Patients and leiomyoma cells}

Fresh tumor samples were obtained from seven individuals at the time of surgery for uterine leiomyoma (median age, 43 years; range: 33-49 years) (Supplementary Table 1). A representative case (75 years old) of uterine lipoleiomyoma was also included as a reference in our morphologic analysis.

Leiomyoma cells were isolated from finely minced or sliced tumor tissue, which was placed in $25 \mathrm{~cm}^{3}$ flasks containing Dulbecco's modified Eagle's medium (DMEM, ThermoFisher Scientific, Yokohama, Japan) supplemented with $10 \%$ fetal bovine serum (FBS) and antibiotics $(100 \mathrm{U} / \mathrm{ml}$ penicillin, $100 \mu \mathrm{g} / \mathrm{ml}$ streptomycin, and $0.25 \mu \mathrm{g} / \mathrm{ml}$ amphotericin B) under standard culture conditions $\left(37^{\circ} \mathrm{C}, 90 \%\right.$ humidified air, $\left.10 \% \mathrm{CO}_{2}\right)$. Harvested and semi-confluent leiomyoma cells $(\sim 90 \%)$ at passages $4-6$ were used in the following analyses. The tumor diagnosis and the exclusion of fat cells in each leiomyoma sample were verified microscopically. Written informed consent was obtained from each patient, and the study design was approved by our institutional review board.

\section{The adipocytic differentiation assay}

The cells were maintained or grown in the above conditions with or without a treatment with $0.1,1$ or $10 \mu \mathrm{mol} / 1$ XAV939 (Sigma-Aldrich Japan, Tokyo, Japan) dissolved in dimethyl sulfoxide (Nacalai Tesque, Kyoto, Japan) [11].
Because serum deprivation can select for marrow-derived mesenchymal progenitor cells with a more pluripotent phenotype [12], the cells were also treated with XAV939 in a medium lacking FBS in 96-well culture plates or culture slides (Corning, NY). In a hypoxic experiment, cells were incubated in a multi-gas incubator $\left(2.5 \% \mathrm{O}_{2}\right.$, APM-300, ASTEC, Fukuoka, Japan). The hypoxic condition was confirmed according to the intracellular oxygen tension, which was tested using a hypoxia-detecting probe, mono azo rhodamine (MAR, Goryo Chemical, Sapporo, Japan), as previously described (Supplementary Figure S1) [13].

After fixation with $10 \%$ neutral buffered formalin for $60 \mathrm{~min}$ at room temperature, the cells were rinsed three times in PBS for $10 \mathrm{~min}$ and stained by oil-red-O solution. Accumulated intracytoplasmic neutral lipids were labeled with $500 \mathrm{nM}$ hydrophilic Nile Red fluorescent dye (AdipoRed, Ronza, Walkersville, MD) and measured using an All-in-One fluorescent microscope imaging system (BZ-X700, Keyence, Osaka, Japan) [14].

\section{Immunohistochemistry}

Leiomyoma cells were fixed with absolute methanol for 60 min followed by three changes of PBS for $5 \mathrm{~min}$ at room temperature, and then incubated with a serum-free blocking reagent (Agilent, Tokyo, Japan) for $10 \mathrm{~min}$. Positive signals were visualized with a secondary antibody-conjugated immunoenzyme polymer reagent (Histofine Simple Stain MAX PO, Nichirei biosciences, Tokyo, Japan) followed by 3,3'-diaminobenzidine tetrahydrochloride (Sigma-Aldrich Japan) or an Alexa Fluor 488 Dye-conjugated anti-mouse secondary antibody (ThermoFisher Scientific) after incubation of the following primary antibodies for $30 \mathrm{~min}$ : anti$\beta$-catenin (14/Beta-Catenin, 1:200, BD Biosciences, Tokyo, Japan), anti- $\alpha$-smooth muscle actin (1A4, 1:300, Agilent), anti-desmin (D33, 1:100, Agilent), and anti-peroxisome proliferator-activated receptor $\gamma$ (PPAR $\gamma$, E-8, 1:200, Santa Cruz Biotechnology, Santa Cruz, CA). In immunohistochemistry for $\beta$-catenin (1:100) using formalin-fixed, paraffin-embedded tumor or myometrial tissues, antigen retrieval in citrate buffer ( $\mathrm{pH}$ 6.0) was performed for $20 \mathrm{~min}$ using a microwave oven. A confocal laser imaging system (LSM880 with Airyscan, Carl Zeiss Microscopy, Tokyo, Japan) was used for the observation of fluorescent signals in $\beta$-catenin immunostaining.

\section{RNA extraction and the real-time RT-PCR}

Total RNA was extracted from leiomyoma cells under a variety of conditions after 2 or 7 days of the treatment using a phenol-based RNA extraction reagent (TRIzol, ThermoFisher Scientific) according to the manufacturer's instructions, and then transcribed into cDNA by SuperScript III reverse transcriptase (ThermoFisher Scientific). A TaqMan 

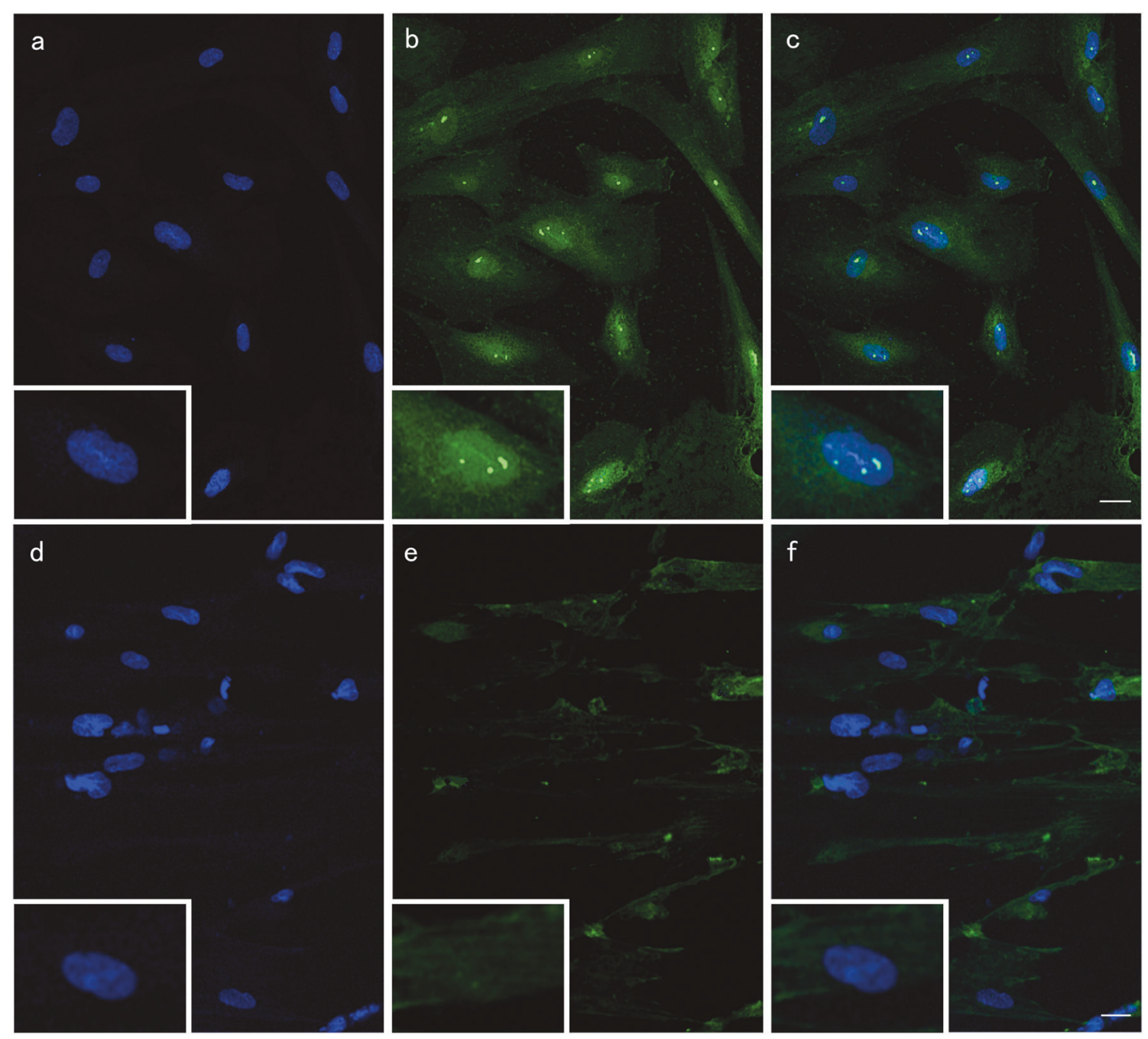

Fig. 1 The effect of XAV939 on the immunohistochemical expression of $\beta$-catenin in human leiomyoma cells. In comparison to non-treated cells $(\mathbf{a}-\mathbf{c})$, the nuclear immunofluorescence of $\beta$-catenin (green; $\mathbf{b}, \mathbf{e})$ was reduced in the $10 \mu \mathrm{mol} / \mathrm{l} \mathrm{XAV}$-treated cells on day $1(\mathbf{d}-\mathbf{f})$.

Gene Expression assay kit (ThermoFisher Scientific) was used for the real-time reverse transcription-polymerase chain reaction (RT-PCR). The mean $\mathrm{Ct}$ value of each sample was measured from tri- or quadruplicate reactions using a StepOnePlus Real-Time PCR system (ThermoFisher Scientific). The relative expression levels of the targeted adipogenic genes, namely those encoding perilipin-1 (PLIN, Hs00605340_m1) and adiponectin (ADIPOQ, Hs00605917_m1), were compared to the succinate dehydrogenase complex subunit A gene (SDHA, Hs00188166_m1) as an endogenous control, using the comparative $\mathrm{Ct}(\Delta \Delta \mathrm{Ct})$ method. To further address the biological function of XAV939 in leiomyoma cells, the expression of the genes encoding myocardin (MYOCD Hs00538076_m1), cyclin D1 (CCND1 Hs00765553_m1), and $\beta$-catenin (CTNNB Hs00355045_m1) was examined.

Nuclear counterstaining was performed with 4',6-diamidino-2-phenylindole (blue) (a, d). Merged images are shown in $\mathbf{c}$ and $\mathbf{f}$. Scale bar $=$ $25 \mu \mathrm{m}$

\section{Cell proliferation assay}

The proliferation of XAV939-treated leiomyoma cells under standard conditions (10\% FBS and normoxia) harvested in a 96-well culture plate was evaluated by quadruplicate measurements at 1,24 , and $48 \mathrm{~h}$ after incubation using a resazurin-based fluorescent reagent (almarBlue ${ }^{\circledR}$, BioSource, Camarillo, CA) and a standard spectrophotometer according to the manufacturer's protocol.

\section{Gene silencing}

Leiomyoma cells were transfected with 50 or $100 \mathrm{nM} \beta$ catenin siRNA (SignalSilence, Cell Signaling Technology, Beverly, MA) for $48 \mathrm{~h}$ according to the manufacturer's instructions. 


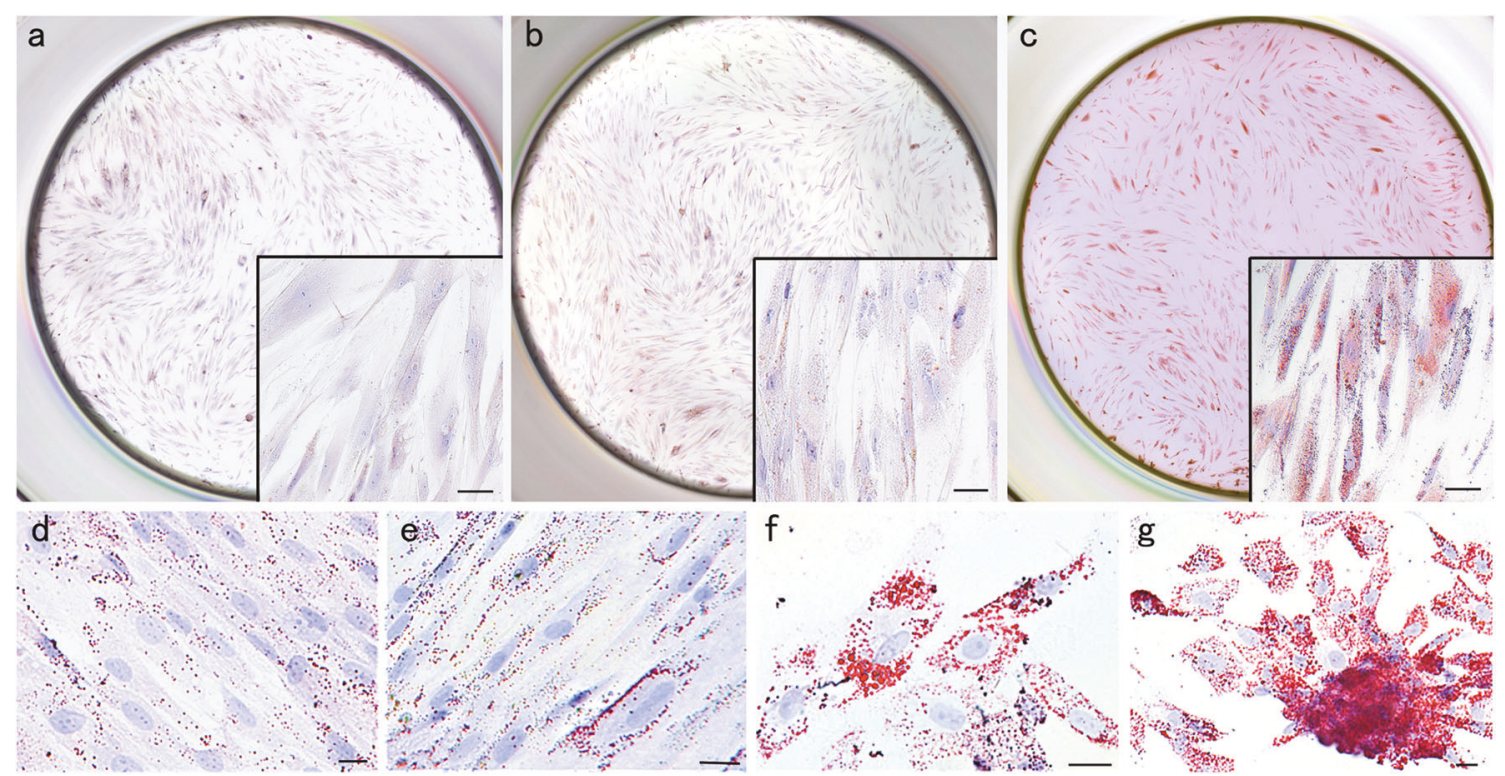

Fig. 2 Lipid accumulation in $1 \mu \mathrm{mol} / \mathrm{l}$ XAV939-treated leiomyoma cells as demonstrated by oil-red-O staining. Although little or only subtle lipid droplet staining was observed in the control (10\% FBS/ normoxia without XAV939) (a) and XAV939-treated/serum-starved cells on day 3 (b), respectively; the treated cells showed abundant fine

\section{Statistical analysis}

The differences between the mean luminance signals and the gene expression values were assessed using the Kruskal-Wallis test or Scheffe's multiple comparison and unpaired Student's $t$-test, respectively. $P$-values of $<0.05$ were considered to indicate statistical significance.

\section{Results}

Leiomyoma cells were initially cultured with various concentrations $(0.1,1$, and $10 \mu \mathrm{mol} / \mathrm{l})$ of XAV939 under standard culture conditions (10\% FBS/normoxia) for 2 weeks. However, lipid droplets were not readily identified by oilred-O staining within this period; however, XAV939 appeared to efficiently inhibit the nuclear localization of $\beta$ catenin in leiomyoma cells (Fig. 1). As serum deprivation is known to awaken mesenchymal pluripotency in stromal cells [12], the cells were treated with XAV939 under a serum-free culture conditions. Thereafter, lipid droplets were detectable and increased in a time-dependent manner (Fig. 2a-c). In addition, the cells were incubated under hypoxic conditions in the subsequent adipogenic induction because hypoxia has been shown to induce adipogenesis in mesenchymal stem cells [15].

Lipid droplets were identified in the cells with oil-red-O after XAV939 treatment in serum-free media under hypoxic conditions on day 1; more lipid droplets accumulated at a lipid droplet staining on day 7 (c) (inset: enlarged view of each well). The lipid accumulation in XAV939-treated/serum-starved cells was enhanced under hypoxic conditions $\left(2.5 \% \mathrm{O}_{2}\right)(\mathbf{d}$ : day 1 , e: day 3, f: day 7 , g: day 14$)$. Scale bar $=25 \mu \mathrm{m}$

later stage (Fig. 2d-g). The findings were consistently observed in the seven cases that were examined, where stably cultured tumor cells were obtained. However, the cells that were treated with $10 \mu \mathrm{mol} / \mathrm{l}$ XAV939 were easily detached from the floors of the culture slides or wellsprobably due to the prominent accumulation of intracytoplasmic lipids and as a consequence of the disturbed $\beta$ catenin function, making it difficult for further observation on day 4 or later. Thus, treatment with $1 \mu \mathrm{mol} / \mathrm{l} \mathrm{XAV939}$ was applied to the following analyses.

The lipid accumulation in XAV939-treated leiomyoma cells was further confirmed and semi-quantified by AdipoRed staining, and was enhanced by serum starvation and much more by additional hypoxic stress (Fig. 3a-c). Under standard culture conditions, the XAV939 treatment did not result in detectable lipid signals (as determined by AdipoRed staining) for at least 7 days. Minimal or insignificant lipid signals were seen in the untreated cells under serumfree and/or hypoxic conditions until day 7 (Fig. 3d). The staining intensity of AdipoRed-stained XAV939-treated cells under the hypoxic and serum-free conditions was increased in a time- and dose-dependent manner (Fig. 3e). The inhibitory role of $\beta$-catenin in adipogenesis was reinforced by the lipid accumulation in leiomyoma cells after the treatment with siRNA against $\beta$-catenin under similar conditions without XAV939 (Supplementary Figure S2). The expression of cyclin D1, a major target of the WNT/ $\beta$ catenin signaling pathway, was reduced by the XAV939 treatment in leiomyoma cells (Supplementary Figure S3). In 
Fig. 3 The lipid accumulation in AdipoRed-stained leiomyoma cells under various conditions on day 7. Minimal, mild, and unequivocal fluorescent signals of AdipoRed were detectable in control cells (10\% FBS/ normoxia without XAV939) (a) and in $1 \mu \mathrm{mol} / \mathrm{l} \mathrm{XAV939-treated}$ cells under normoxic (b) and hypoxic (c) conditions with serum-free media, respectively. Scale bar $=25 \mu \mathrm{m}$. The mean \pm $\mathrm{SD}$ of relative AdipoRed luminance of the cells (determined by pentaplicate measurements) is shown (d). C control (normoxia and $10 \%$ FBS), H hypoxic condition, NS non-serum, X XAV939-treated. $* P<0.01, * * P<0.001$ by Scheffe's multiple comparison. The luminance of AdipoRed signals in the XAV939 ( 0.1 or 1 $\mu \mathrm{M})$-treated cells under hypoxic and serum-free conditions was increased in a time- and dosedependent manner (e). $* P=$ 0.0244 by Kruskal-Wallis test
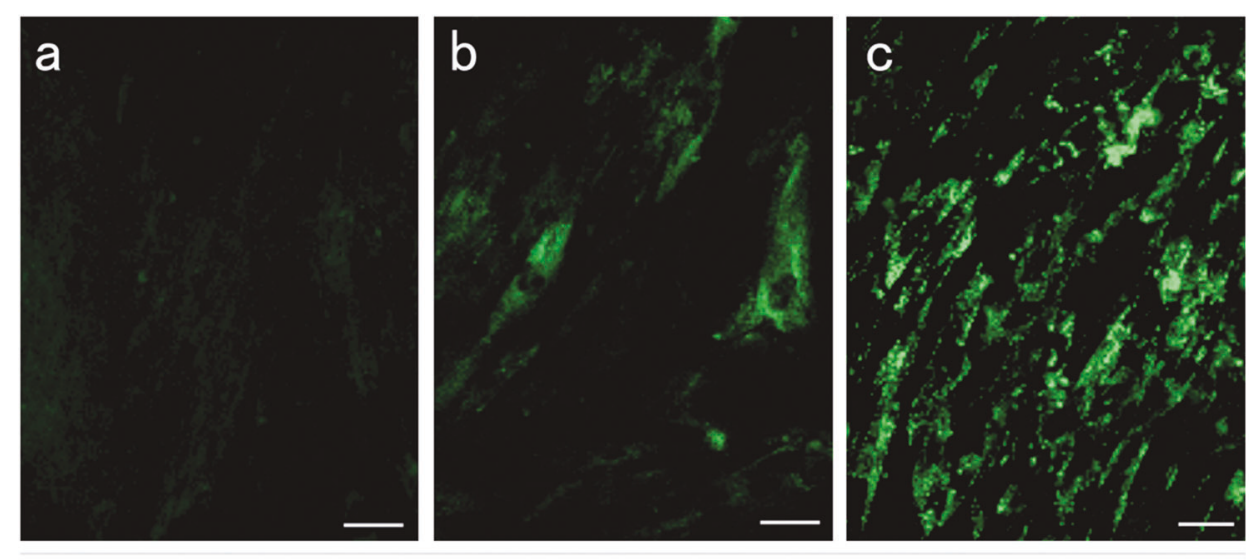

d

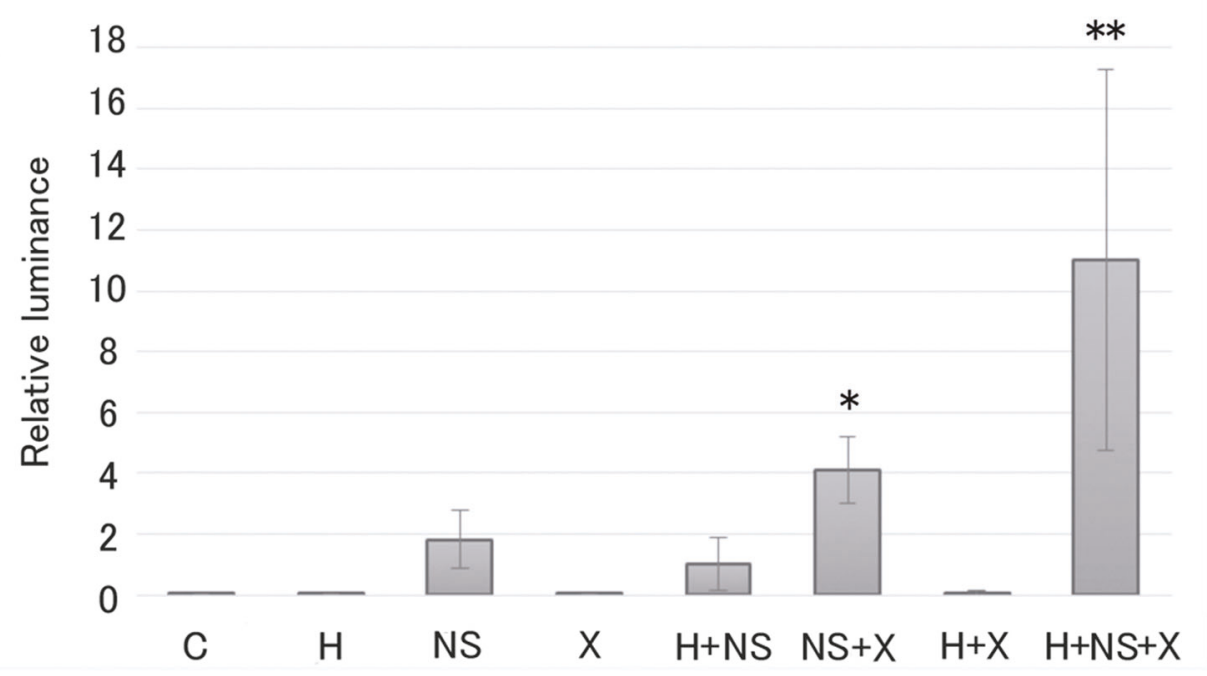

e

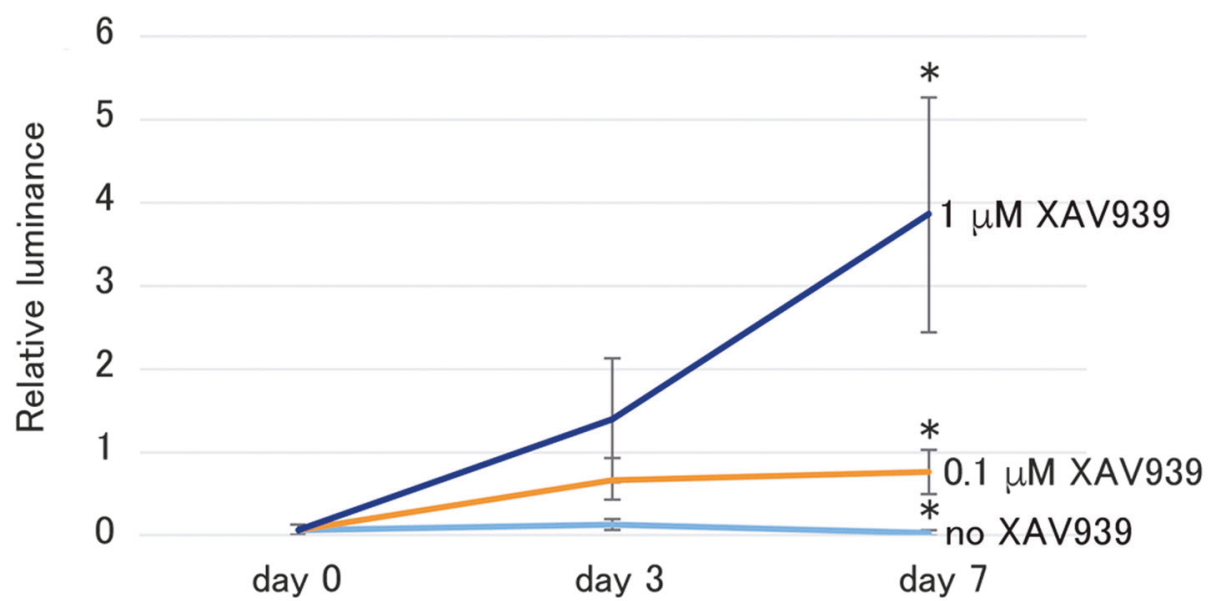

addition, the XAV939 treatment was able to suppress the cell proliferation in a dose-dependent manner (Supplementary Figure S4).
To further characterize the leiomyoma cells with lipid accumulation, the expression of adipogenic markers (PPAR $\gamma$, adiponectin and perilipin-1) was assessed 


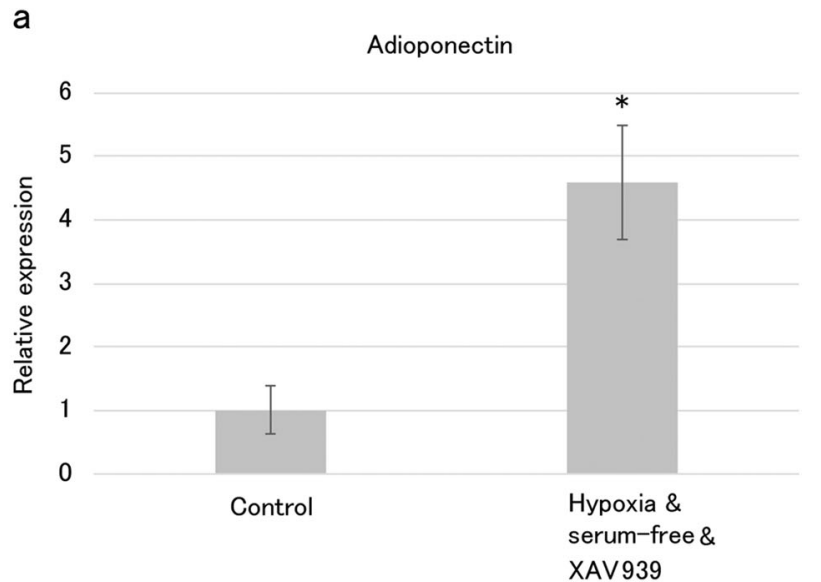

b

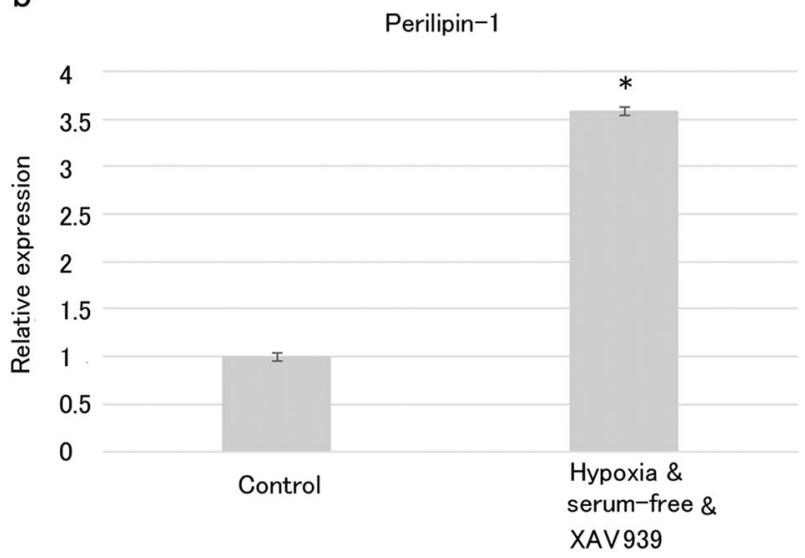

Fig. 4 The relative expression of adipocytic differentiation markers in leiomyoma cells obtained from a case. Transcripts of adiponectin (a) and perilipin-1 (b) are highly expressed in cells treated with XAV939 under hypoxia and with serum starvation in comparison to cells without adipocytic induction (control) on day 7. $* P<0.001$

immunohistochemically and/or semi-quantitatively at the mRNA level. The real-time RT-PCR revealed that after 7 days of the above adipocytic induction (serum-free/ hypoxia/XAV939) the mRNAs of adiponectin and perilipin-1 were more highly expressed in leiomyoma cells in comparison to control cells incubated under normoxic conditions with a medium containing 10\% FBS and no XAV939 (Fig. 4). In contrast, the expression of PPAR $\gamma$ was detected in both the treated and non-treated cells, or was rather reduced in the former (Fig. 5). With regard to the smooth muscle phenotype, the immunohistochemical expression of desmin was suppressed in the cells after the adipocytic induction (Fig. 6a-c), while that of $\alpha$-smooth muscle actin was mostly well-preserved (Fig. 6d-f). However, the mRNA expression of the MYOCD that encodes a potent smooth/cardiac muscle-specific transcriptional coactivator, directing cell differentiation towards smooth muscle,

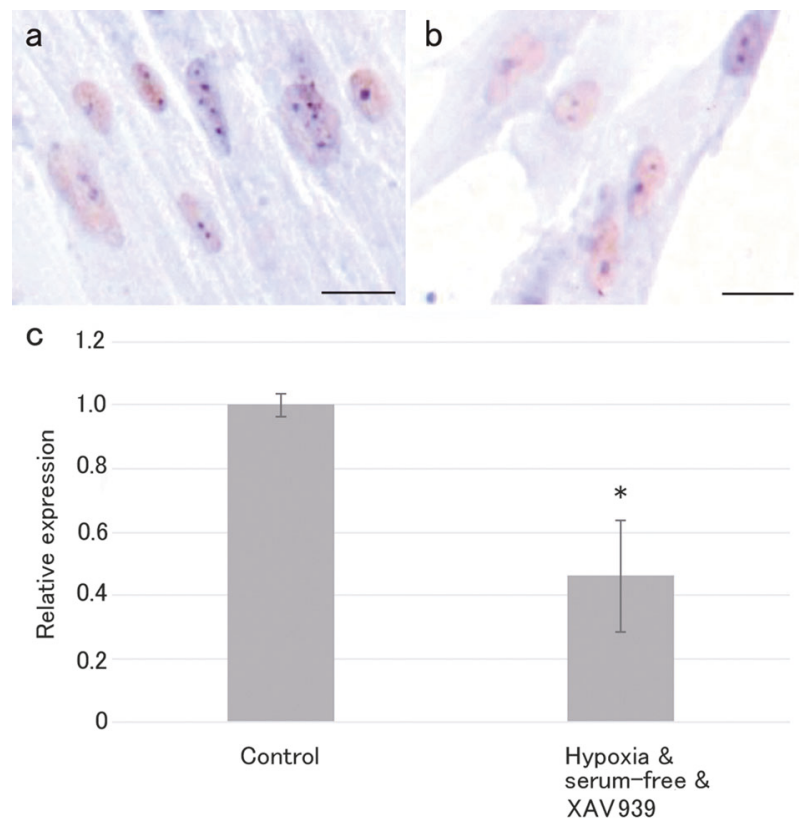

Fig. 5 The expression of PPAR $\gamma$ in leiomyoma cells obtained from a case. The nuclear immunolocalization of PPAR $\gamma$ was seen in cells on day 7 with (a) or without (b) adipocytic induction (hypoxia/serumfree/XAV939). Scale bar $=25 \mu \mathrm{m}$. The real-time RT-PCR revealed that the relative expression of PPAR $\gamma$ was reduced in cells with adipocytic induction $(\mathbf{c}) .{ }^{*} P<0.05$

was not downregulated after the adipocytic induction (Supplementary Figure S5).

In the lipoleiomyoma examined (Fig. 7a-d), a relatively small number of spindle-shaped smooth muscle cells were sparsely distributed among vacuolated mature-looking fat cells. Immunohistochemically, the smooth muscle cells were unequivocally positive for desmin but only faintly positive for $\beta$-catenin, whereas mild but often diffuse expression of $\beta$-catenin, which was mostly in an intracytoplasmic manner, was seen in myometrial smooth muscle and leiomyoma (Fig. 7e, f; Supplementary Table 1).

\section{Discussion}

In contrast to leiomyoma, which is the most common uterine mesenchymal neoplasm, smooth muscle tumors with a fatty component, referred to as lipoleiomyomas, are relatively rare, accounting for $<2.9 \%$ of all uterine leiomyomas [1, 2]. The proportion of the fatty component within the lesion is highly variable and may be shown as an almost entirely fatty neoplasm simulating lipoma. Several pathogenetic mechanisms underlying the presence of fat cells within leiomyoma have been discussed previously $[3-5,16]$. Although the direct transformation (metaplasia or 


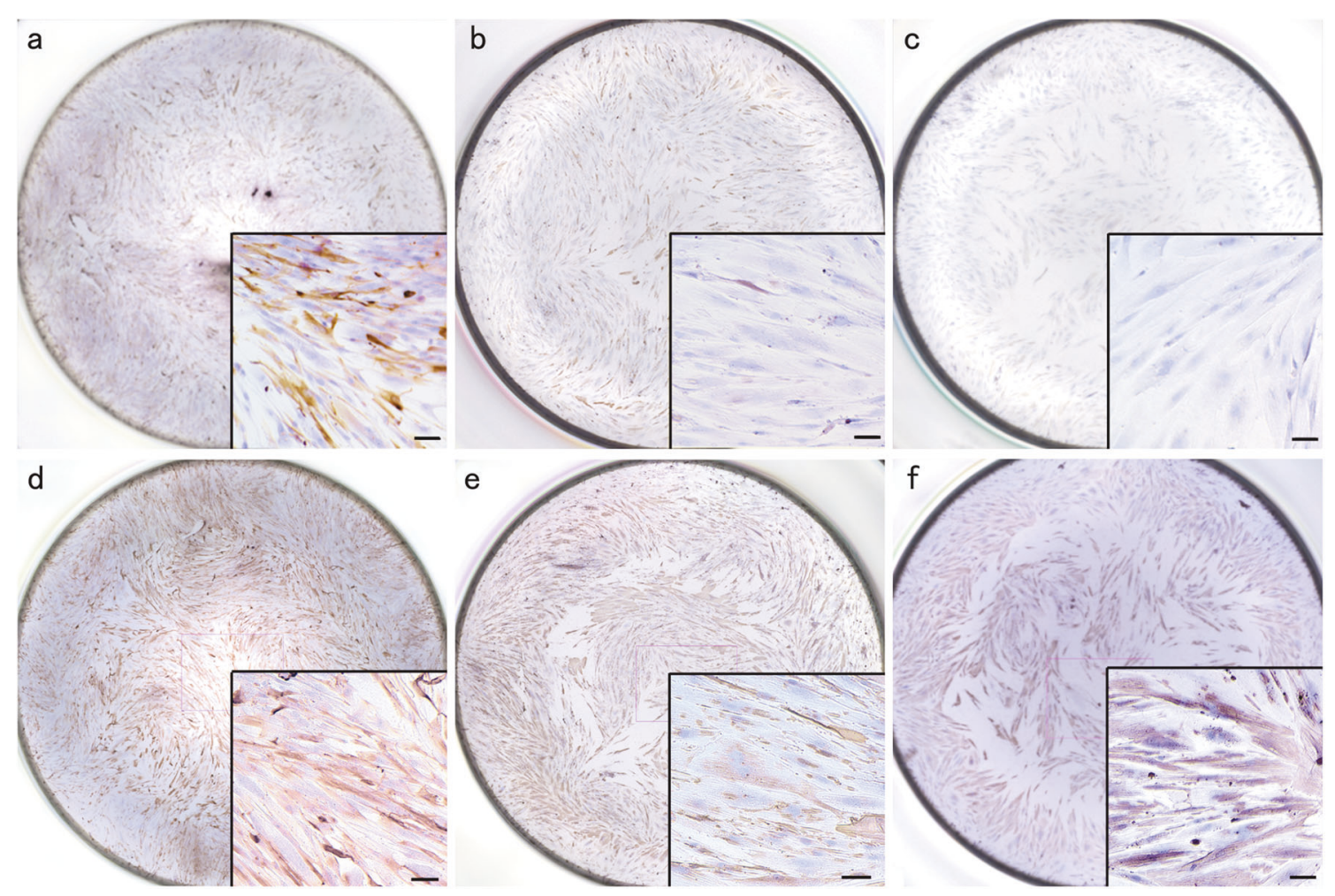

Fig. 6 Phenotypic changes in the smooth muscle differentiation of XAV939-treated leiomyoma cells under non-serum and hypoxic conditions. The immunohistochemical expression of desmin $(\mathbf{a}-\mathbf{c})$ and $\alpha$-smooth muscle actin $(\mathbf{d}-\mathbf{f})$ are depicted in low-power and enlarged (insets) views of leiomyoma cells grown in a 96-well culture plate at 1

transdifferentiation) of smooth muscle cells into adipocytes is a plausible hypothesis, little attention has been paid to the phenotypic plasticity of smooth muscle cells in vivo or in vitro.

Our study demonstrated that adipocytic transdifferentiation, as shown by intracellular lipid accumulation and the enhanced expression of adipogenic markers (i.e., adiponectin, perilipin-1), can be elicited in cultured uterine leiomyoma cells by XAV939 treatment together with serum starvation and hypoxia. However, PPAR $\gamma$, a representative transcriptional regulator of adipogenesis, was downregulated by the treatment on day 7 . It is difficult to explain the somewhat discordant expression pattern of adipogenic markers that was observed in our model. The hypoxiainduced downregulation of PPAR $\gamma$ has been described in smooth muscle cells of the pulmonary artery [17]. In addition, PPAR $\gamma$ is highly expressed in uterine leiomyoma [18]. When the critical role of PPAR $\gamma$ in the early phase of adipogenesis is also taken into consideration [19], our observation of the PPAR $\gamma$ expression might not be surprising.

Notably, the phenotypic switch of leiomyoma cells can be initiated without the addition of any known adipocyteinducing or differentiation-inducing supplements (i.e., insulin and dexamethasone) to the culture media using our (a, d), 3 (b, e), and 7 (c, f) days after XAV939 treatment with serum starvation and hypoxia. Scale bar $=25 \mu \mathrm{m}$

model. Although adipocytic differentiation has been described in human vascular smooth muscle cells in vitro, they were cultured in a standard adipocyte differentiation medium supplemented with insulin, dexamethasone, triiodothyronine, and 3-isobutyl-1-methylxanthine [20].

The WNT signaling transduced by $\beta$-catenin is known to antagonize adipogenesis by inhibiting the functions of several adipogenic transcription factors, including PPAR $\gamma$ and CCAAT/enhancer binding protein- $\alpha$ in preadipocytes or mesenchymal stem cells [6, 7]. In addition, WNT signaling has a role in the maintenance of the smooth muscle phenotype and exerts cellular proliferation in uterine leiomyoma [11]. Our study, which used XAV939-a potent inhibitor of $\beta$-catenin-was conducted to investigate these scenarios. The results suggested that the inhibition of $\beta$ catenin allows leiomyoma cells to reach a state in which cellular proliferation is terminated and in which cells are directed towards an adipocytic lineage. Although the expression of $\beta$-catenin in formalin-fixed, paraffinembedded leiomyoma or peritumoral myometrium tissues was only mildly immunohistochemically detectable (predominantly in an intracytoplasmic manner), that in spindle tumor cells and fat cells was faintly detectable or mostly lacking in the lipoleiomyomas in our study. However, the native 

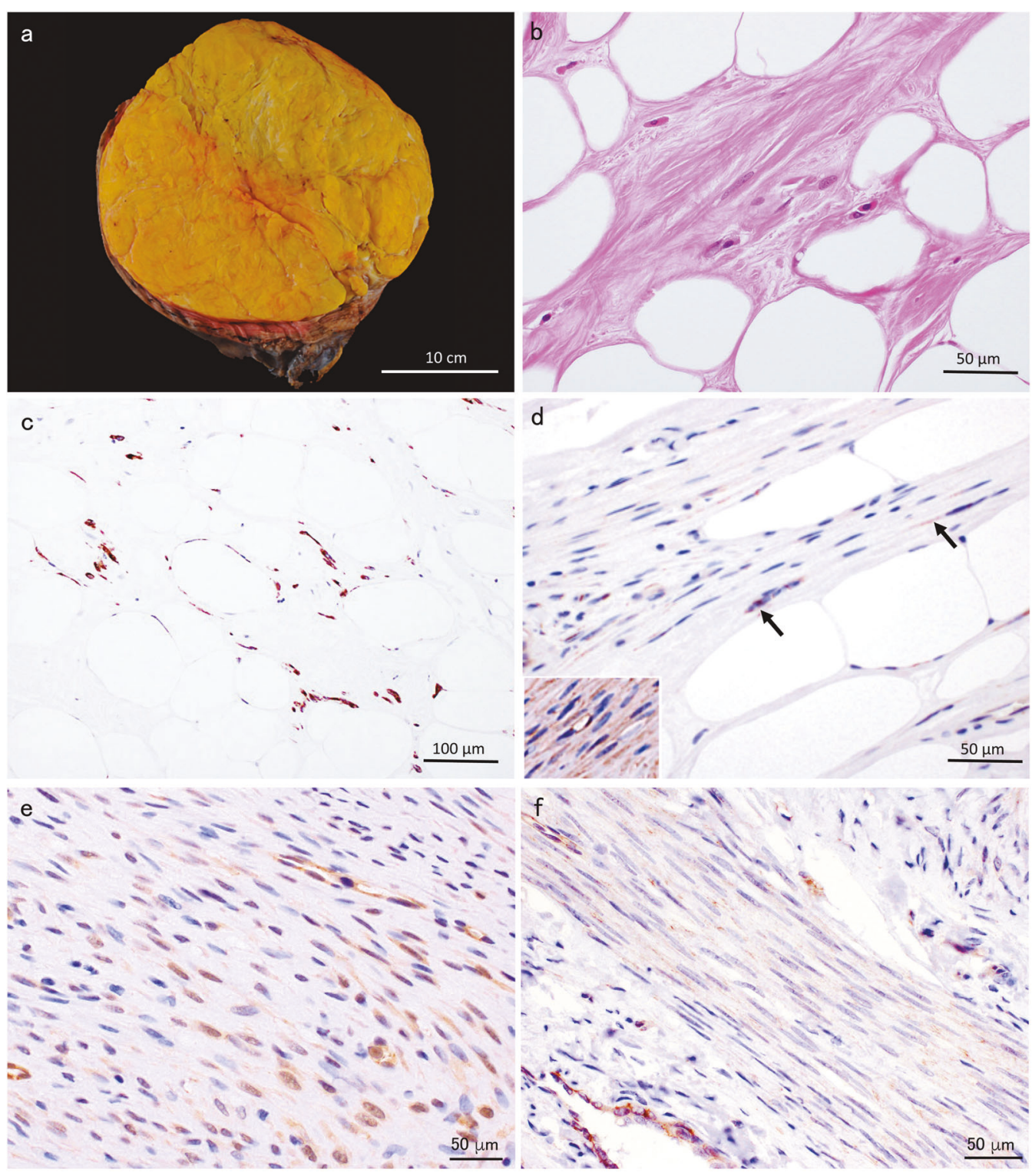

Fig. 7 A huge lipoleiomyoma with a prominent lipomatous element arising in the uterus of an elderly woman. The entire cut surface of the tumor had a yellowish lipomatous appearance (a). Spindle-shaped smooth muscle cells were sparsely distributed among vacuolated mature-looking fat cells $(\mathrm{H} \& \mathrm{E})(\mathbf{b})$. The spindle-shaped smooth muscle

cells were immunoreactive to desmin (c) and only faintly immunoreactive to $\beta$-catenin (arrows, inset: peritumoral myometrium) (d). Mild nuclear (e) and/ or intracytoplasmic $(\mathbf{e}, \mathbf{f})$ immunohistochemical expression of $\beta$-catenin was seen in leiomyoma (e) and peritumoral myometrium (f).

molecules or stimuli that can inhibit or antagonize $\beta$-catenin in uterine lipoleiomyomas remain to be identified in future studies.

It is also notable that the adipocytic transdifferentiation in leiomyoma cells was induced by the inhibition of $\beta$ catenin combined with serum starvation and further enhanced by hypoxic stress in the current study. However, how serum starvation and hypoxia can modulate the functions of the $\mathrm{WNT} / \beta$-catenin signaling or promote cell potency to aberrantly differentiate remains elusive. A recent study demonstrated that the combined use of a small molecule (KY02111) and other WNT signaling modulators such as XAV939 can induce the robust cardiac

differentiation of serum-starved human pluripotent stem cells [21]. Moreover, serum deprivation has been shown to result in the downregulation of the $\mathrm{WNT} / \beta$-catenin signaling in cancer cells by upregulated Wnt3a leading to the internalization of low-density lipoprotein receptor-related protein 6 , which reduces the nuclear accumulation of $\beta$ catenin [22]. The hypoxia-induced downregulation of $\beta$ catenin has been also described in human cancer cells [23, 24]. Thus, serum starvation and hypoxia might have functioned cooperatively in the further inhibition of WNT/ $\beta$ catenin signaling, leading to the alteration in the differentiation of the current XAV939-treated leiomyoma cells. 
Standard culture conditions using media supplemented with $10 \%$ FBS in ambient air do not seem to reflect the in vivo milieu, and the microenvironment of the myometrium or leiomyoma is physiologically relevant to such harsh conditions [25, 26]. Myometrial contraction and vasoconstriction during the menstrual cycle can result in repeated hypoxia in the uterine wall and leiomyoma. Low-oxygen tension is known to be an important component of stem cell niche and to provide signals that maintain the stem cell functions [27, 28], and a hypoxic state is essential for early embryogenesis or variable organogenesis, such as that observed in cardiovascular development [29]. Besides, serum deprivation can select more pluripotent populations from bone marrow- or adipose tissue-derived stem cells $[12,30]$. Various mesenchymal stem cells and progenitor cells cultured under hypoxic conditions have an enhanced ability to differentiate into adipocytes $[15,31,32]$. Hypoxia and serum starvation may therefore induce leiomyoma cells to transform into a progenitor cell phenotype that is susceptible to the subsequent adipocytic differentiation exerted by the suppressed $\beta$-catenin function.

The mechanisms involved in the switch in the differentiation of leiomyoma cells induced by the WNT signaling blockade under this harsh in situ microenvironment may also be related to the development of distinct or heterologous tissue elements in some unusual mesenchymal tumors, including parosteal lipoma (lipoma with an osteocartilagineous element), myolipoma (lipoma with a smooth muscle element), and so-called cutaneous angiomyolipoma (angioleiomyoma with a fatty element). It may be worth examining the WNT/ $\beta$-catenin signaling status as well as the stress microenvironments in these tumors to address this unique process of cellular metamorphosis.

In conclusion, adipocytic transdifferentiation can be induced in uterine leiomyoma cells by treatment with a $\beta$ catenin inhibitor under serum starvation conditions and is much enhanced by additional hypoxia, suggesting the cooperative or synergistic roles of these stresses in a potential switch in differentiation from smooth muscle cells to adipocytes. Our model may support that the inhibition of $\mathrm{WNT} / \beta$-catenin signaling is essentially involved in the development of lipoleiomyoma.

\section{Compliance with ethical standards}

Conflict of interest The authors declare that they have no conflict of interest.

\section{References}

1. Aung $\mathrm{T}$, Goto $\mathrm{M}$, Nomoto $\mathrm{M}$, et al. Uterine lipoleiomyoma: a histopathological review of 17 cases. Pathol Int. 2004;54:751-8.

2. Akbulut M, Gündoğan M, Yörükoğlu A. Clinical and pathological features of lipoleiomyoma of the uterine corpus: a review of 76 cases. Balk Med J. 2014;31:224-9.
3. Jones $\mathrm{C}$, Shalin SC, Gardner JM. Incidence of mature adipocytic component within cutaneous smooth muscle neoplasms. J Cutan Pathol. 2016;43:866-71.

4. Lin M, Hanai J. Atypical lipoleiomyoma of the uterus. Acta Pathol Jpn. 1991;41:164-9.

5. Chibisheva V, Antovska V, Trajanova M, et al. Vascular lipoleiomyoma of the uterus: an unusual case. Med Arch. 2016;70:473-6.

6. Ross SE, Hemati N, Longo KA, et al. Inhibition of adipogenesis by Wnt signaling. Science. 2000;289:950-3.

7. Takada I, Kouzmenko AP, Kato S. Wnt and PPAR $\gamma$ signaling in osteoblastogenesis and adipogenesis. Nat Rev Rheumatol. 2009;5:442-7.

8. Arango NA, Szotek PP, Manganaro TF, et al. Conditional deletion of $\beta$-catenin in the mesenchyme of the developing mouse uterus results in a switch to adipogenesis in the myometrium. Dev Biol. 2005;288:276-83.

9. Huang S-MA, Mishina YM, Liu S, et al. Tankyrase inhibition stabilized axin and antagonizes Wnt signaling. Nature. 2009;461:614-20.

10. Zhou S, Yi T, Shen K, et al. Hypoxia: The driving force of uterine myometrial stem cells differentiation into leiomyoma cells. Med Hypotheses. 2011;77:985-6.

11. Ono M, Yin P, Navarro A, et al. Inhibition of canonical WNT signaling attenuates human leiomyoma cell growth. Fertil Steril. 2014;101:1441-9.

12. Pochampally RR, Smith JR, Ylostalo J, et al. Serum deprivation of human marrow stromal cells (hMSCs) selects for a subpopulation of early progenitor cells with enhanced expression of OCT-4 and other embryonic genes. Blood. 2004;103:1647-52.

13. Piao W, Tsuda S, Tanaka Y, et al. Development of azo-based fluorescent probes to detect different levels of hypoxia. Angew Chem Int Ed Engl. 2013;52:13028-32.

14. Shilpa K, Dinesh T, Lakshmi BS. An in vitro model to probe the regulation of adipocyte differentiation under hyperglycemia. Diabetes Metab J. 2013;37:176-80.

15. Fink T, Abildtrup L, Fogd K, et al. Induction of adipocyte-like phenotype in human mesenchymal stem cells by hypoxia. Stem Cells. 2004;22:1346-55.

16. Resta L, Maiorano E, Piscitelli D, et al. Lipomatous tumors of the uterus. Clinico-pathological features of 10 cases with immunocytochemical study of histogenesis. Pathol Res Pract. 1994;190:378-83.

17. Lu X, Bijli KM, Ramirez A, et al. Hypoxia downregulates PPAR $\gamma$

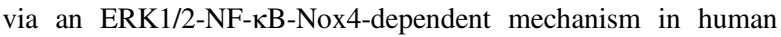
pulmonary artery smooth muscle cells. Free Radic Biol Med. 2013;63:151-60.

18. Tsibris JCM, Porter KB, Jazayeri A, et al. Human uterine leiomyomata express higher levels of peroxisome proliferatoractivated receptor $\gamma$, retinoid receptor $\alpha$, and all-trans retinoic acid than myometrium. Cancer Res. 1999;59:5737-44.

19. Tzameli I, Fang H, Ollero M, et al. Regulated production of a peroxisome proliferator-activated receptor- ligand during an early phase of adiposcyte differentiation in 3T3-L1 adipocytes. J Biol Chem. 2004;279:36093-102.

20. Davies JD, Carpenter KLH, Challis IR, et al. Adipocytic differentiation and liver $\mathrm{X}$ receptor pathways regulate the accumulation of triacylglycerols in human vascular smooth muscle cells. J Biol Chem. 2005;280:3911-9.

21. Minami I, Yamada K, Otsuji TG, et al. A small molecule that promotes cardiac differentiation of human pluripotent stem cells under defined, cytokine- and xeno-free conditions. Cell Rep. 2012;2:1448-60.

22. He Q, Yan H, Liu J, et al. Wnt3a suppresses Wnt/ $\beta$-catenin signaling and cancer cell proliferation following serum deprivation. Exp Cell Res. 2016;341:32-41. 
23. Wang D, Wang $\mathrm{Y}$, Kong $\mathrm{T}$, et al. Hypoxia-induced $\beta$-catenin downregulation involves p53-dependent activation of Siah-1. Cancer Sci. 2011;102:1322-8.

24. Scholten DJ II, Timmer CM, Peacock JD, et al. Downregulation of Wnt signaling mitigates hypoxia-induced chemoresistance in human osteosarcoma cells. PLoS ONE. 2014;9:e111431.

25. Mayer A, Höckel M, Wree A, et al. Lack of hypoxic response in uterine leiomyomas despite severe tissue hypoxia. Cancer Res. 2008;68:4719-26.

26. Bonazza C, Andrade SS, Sumikawa T, et al. Primary human uterine leiomyoma cell culture quality control: some properties of myometrial cells cultured under serum deprivation conditions in the presence of ovarian steroids. PLoS ONE. 2016;11:e0158578.

27. Choi JR, Pingguan-Murphy B, Wan Abas WA, et al. Impact of low oxygen tension on stemness, proliferation and differentiation potential of human adipose-derived stem cells. Biochem Biophys Res Commun. 2014;448:218-24.
28. Mohyeldin A, Garzon-Muvdi T, Quinones-Hinojosa A. Oxygen in stem cell biology: a critical component of the stem cell niche. Cell Stem Cell. 2010;7:150-61.

29. Webster WS, Abela D. The effect of hypoxia in development. Birth Defects Res C Embryo Today. 2007;81:215-28.

30. Heneidi S, Simerman AA, Keller E, et al. Awakened by cellular stress: isolation and characterization of a novel population of pluripotent stem cells derived from human adipose tissue. PLoS ONE. 2013;8:e64752.

31. Karahuseyinoglu S, Kocaefe C, Balci D, et al. Functional structure of adipocytes differentiated from human umbilical cord stromaderived stem cells. Stem Cells. 2008;26:682-91.

32. Itoigawa $\mathrm{Y}$, Kishimoto $\mathrm{KN}$, Okuno $\mathrm{H}$, et al. Hypoxia induces adipogenic differentiation of myoblastic cell lines. Biochem Biophys Res Commun. 2010;399:721-6. 